\title{
NECROSIS PAPILAR RENAL: EL DIAGNÓSTICO DE UN CLÁSICO EN LA ERA DE LA TOMOGRAFÍA MUITIIDETECTOR
}

\author{
Drs. Leonardo Lidid $A^{(1)}$, Mario Mora $V^{(2)}$, Tamara Ramírez $P^{(3)}$.
}

1. Profesor Asistente de Radiología. Campus Occidente, Facultad de Medicina, Universidad de Chile, Hospital San Juan de Dios.

2. Residente de Radiología. Campus occidente, Facultad de Medicina, Universidad de Chile,

Hospital San Juan de Dios.

3. Médico-Cirujano. Facultad de Medicina, Universidad de Chile.

RENAL PAPILLARY NECROSIS: THE DIAGNOSIS OF A CLASSIC IN THE ERA OF THE MULTISLICE COMPUTED TOMOGRAPHY

\begin{abstract}
Renal papillary necrosis is a multifactorial entitiy that encompasses a wide range of consecuences, including end-stage renal impairment or even death. Its appearance on intravenous pyelography pictures reveals multiple patterns of papillary excavation, fairly well defined in traditional literature. With the advent of multislice computed tomography urography these findings have been refined and new radiological signs such as detection of early renal medullary changes, among others, have been added. The application of this imaging modality may translate into significant short-term improvements in the evolution and prognosis of these patients. This paper is intended to provide both a reviewing and an updating of renal papillary necrosis imaging issues.

Keywords: Abscess, Kidney papillary necrosis, Nephrocalcinosis, Pyelonephritis, Renal medullary necrosis, Tuberculosis.
\end{abstract}

Resumen: La necrosis papilar renal es una condición originada por múltiples factores, con consecuencias variadas incluyendo dentro de ellas el desarrollo de insuficiencia renal terminal y eventualmente la muerte. Su presentación en la pielografía de eliminación demuestra múltiples patrones de excavación papilar, que están muy bien descritos en la literatura clásica.

Lidid L y cols. Necrosis papilar renal: El diagnóstico de un clásico en la era de la tomografía multidetector. Rev Chil Radiol 2010; 16(3): 128-133.

Correspondencia: Dr. Leonardo Lidid A.

Facultad de Medicina. Campus Occidente. Universidad de Chile. Casilla 33052-correo 33. Santiago.

leolidid@gmail.com

Trabajo recibido el 05 de julio de 2010, aceptado para publicación el 23 de agosto de 2010.
Con el advenimiento de la urografía por tomografía computada multicorte, estos hallazgos han sido refinados, agregándose también nuevos signos, que incluyen, entre otros, la detección de cambios medulares precoces que podrían implicar, en un futuro cercano, un significativo cambio en la evolución y pronóstico de estos pacientes. En esta publicación hacemos una revisión y puesta al día de los aspectos imaginológicos de la necrosis papilar renal.

Palabras clave: Absceso, Necrosis medular renal, Necrosis renal papilar, Nefrocalcinosis, Pielonefritis, Tuberculosis.

\section{Introducción}

La necrosis papilar renal (NPR) no se define como una entidad patológica sino como un término descriptivo para una condición multifactorial, en que muchas veces coexiste una variedad de causas posibles; entre las más conocidas se incluyen: diabetes, abuso de antiinflamatorios no esteroidales (AINES), pielonefritis, tuberculosis, trombosis de la vena renal, rechazo de trasplante renal, anemia de células falciformes y uropatía obstructiva. Otras raras causas de NPR son: síndrome diarreico infantil severo, necrosis tubular aguda y alcoholismo ${ }^{(1)}$. Tradicionalmente, en la diabetes se describía una forma fulminante asociada a infección urinaria, obstrucción urinaria y muerte. Sin embargo, en la actualidad, tal vez derivado del mejor control de las infecciones, este cuadro tan violento ya no se ve con frecuencia, observándose más bien otras presentaciones de la enfermedad: la forma crónica bilateral y los cambios isquémicos precoces ${ }^{(1,2)}$.

Entre las consecuencias inmediatas de la necrosis papilar aguda (NPA) se describen el deterioro en la función renal y el síndrome nefrótico ${ }^{(3)}$. Si bien no existe un tratamiento específico, tradicionalmente se ha considerado que el control de las causas desencadenantes puede evitar la progresión de la enfermedad ${ }^{(4)}$. Este concepto ha evolucionado, observándose que la detección precoz que posibilita 
la tomografía computada multidetector (TCMD) y que no era posible con los métodos tradicionales de imagen, abre la posibilidad de no sólo de detener sino eventualmente de revertir la lesión, antes que ésta se consolide ${ }^{(5)}$.

En la NPA, clásicamente diagnosticada por urografía endovenosa, el uso de tomografía computada (TC) contrastada y urografía por tomografía computada (UroTC) puede demostrar una más amplia variedad de alteraciones, dentro de las que se incluyen diferentes tipos de excavaciones llenas de contraste en la médula renal, lesiones no captantes rodeadas de medio de contraste excretado y calcificaciones medulares ${ }^{(1)}$. Asimismo, un defecto de llene en la pelvis renal puede corresponder a una papila desprendida, por lo que una minuciosa observación del complejo papilo-calicial nos puede ayudar en el diagnóstico diferencial entre coágulos, tumores, litiasis y otras causas ${ }^{(4)}$.

En esta revisión nos enfocaremos en las diversas presentaciones de esta enfermedad en UroTC, haciendo especial énfasis en el diagnóstico diferencial y en los nuevos aportes que ha realizado este método de estudio al diagnóstico y pronóstico de esta patología.

\section{Anatomía y fisiopatología de la necrosis papilar}

El sistema colector renal se origina en la corteza renal, donde el primer filtrado urinario entra en la cápsula glomerular para luego pasar a los túbulos colectores renales, que se fusionan y forman ductos colectores que atraviesan la médula para luego vaciarse en la punta de la pirámide medular, llamada papila renal. El número de papilas renales es muy variable, desde 4 hasta 18, con un promedio de 7 a 9 en un riñón típico; cada una de éstas es sostenida por un correspondiente cáliz menor, que recibe el flujo de los ductos colectores.

La médula y la papila renal son particularmente vulnerables a la hipoxia, por la particular distribución de su irrigación y el medio hipertónico que las envuelve. Incluso en individuos saludables existe una hipoxia relativa secundaria al lento flujo sanguíneo en los vasa recta, por lo que condiciones que reducen el flujo sanguíneo pueden producir franca necrosis isquémica ${ }^{(1,4)}$.

\section{Signos radiológicos clásicos}

Los hallazgos radiológicos clásicos de necrosis papilar fueron descritos inicialmente en la urografía endovenosa. Actualmente estos signos también pueden demostrarse, al menos con la misma calidad de imagen, en la fase excretora de la UroTC, agregándose como ventaja con este último método, la posibilidad de utilizar cortes finos y reconstrucciones multiplanares que permiten analizar hallazgos finos y conseguir una completa evaluación de los cálices, hecho que no era posible en la urografia excretora clásica, dado que no siempre se conseguía una total desproyección de ellos ${ }^{(1,6)}$.

El diagnóstico radiológico de la NPR se basa en la demostración de las alteraciones del complejo papilo-calicial con pérdida focal de parénquima renal. Estas alteraciones se pueden dividir, según su especificidad, en dos grupos (Figura 1) ${ }^{(4)}$.

\section{Signos característicos de necrosis papilar:}

- Excavación papilar central: "huevo en copa", también llamado "ball in tee"(7)

- Excavación fornicial unilateral ó bilateral ("Iobster claw")

- Sombra en anillo o anillo de sello ("signet ring").

2. Signo no específico:

- Cálices en "porra" o en "palillo de tambor" ("clubbed calix")(4).

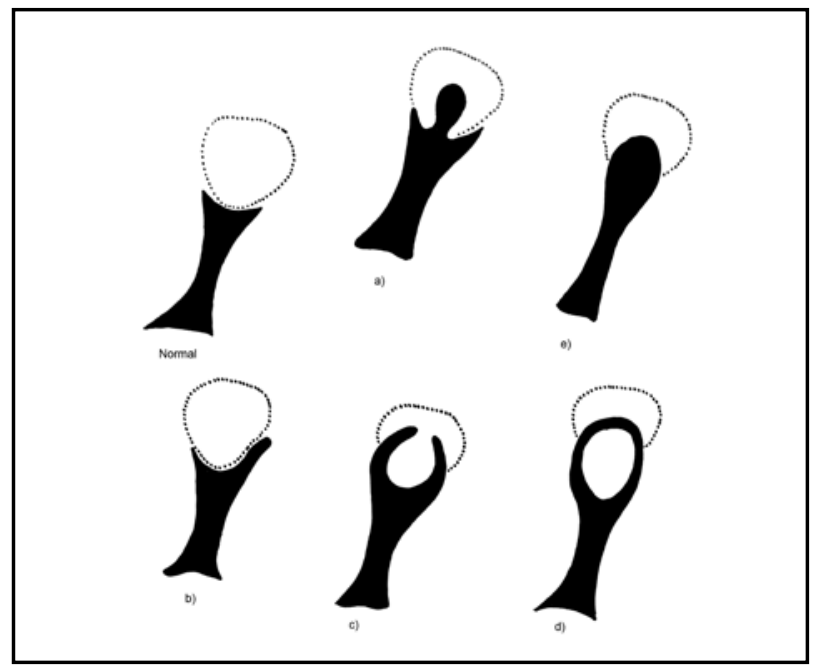

Figura 1. Diferentes patrones de excavaciones con contraste en NPR. a) Huevo en copa "ball in tee". b) Excavación forniceal unilateral. c)bilateral ("lobster claw"). d) Sombra en anillo ("signet ring"). e) Cáliz en "porra" ("Clubbed calix"). Fila superior: formas medulares. Fila inferior: formas papilares.

Además de esta forma de clasificación, estos signos han sido clásicamente divididos en tres formas:

- La forma medular: la excavación se produce en la punta de la papila dando la clásica apariencia llamada, "huevo en copa" o también conocida en lenguaje anglosajón como "ball in tee" (literalmente balón en pedestal) (Figura 2). El desprendimiento papilar comienza en la porción central del cáliz formando una cavidad redonda u oval (balón) ${ }^{(1)}$.

- La forma papilar: se produce necrosis de una gran porción de la papila, que comienza en los fórnices caliciales y el defecto resultante generalmente es de forma triangular. 

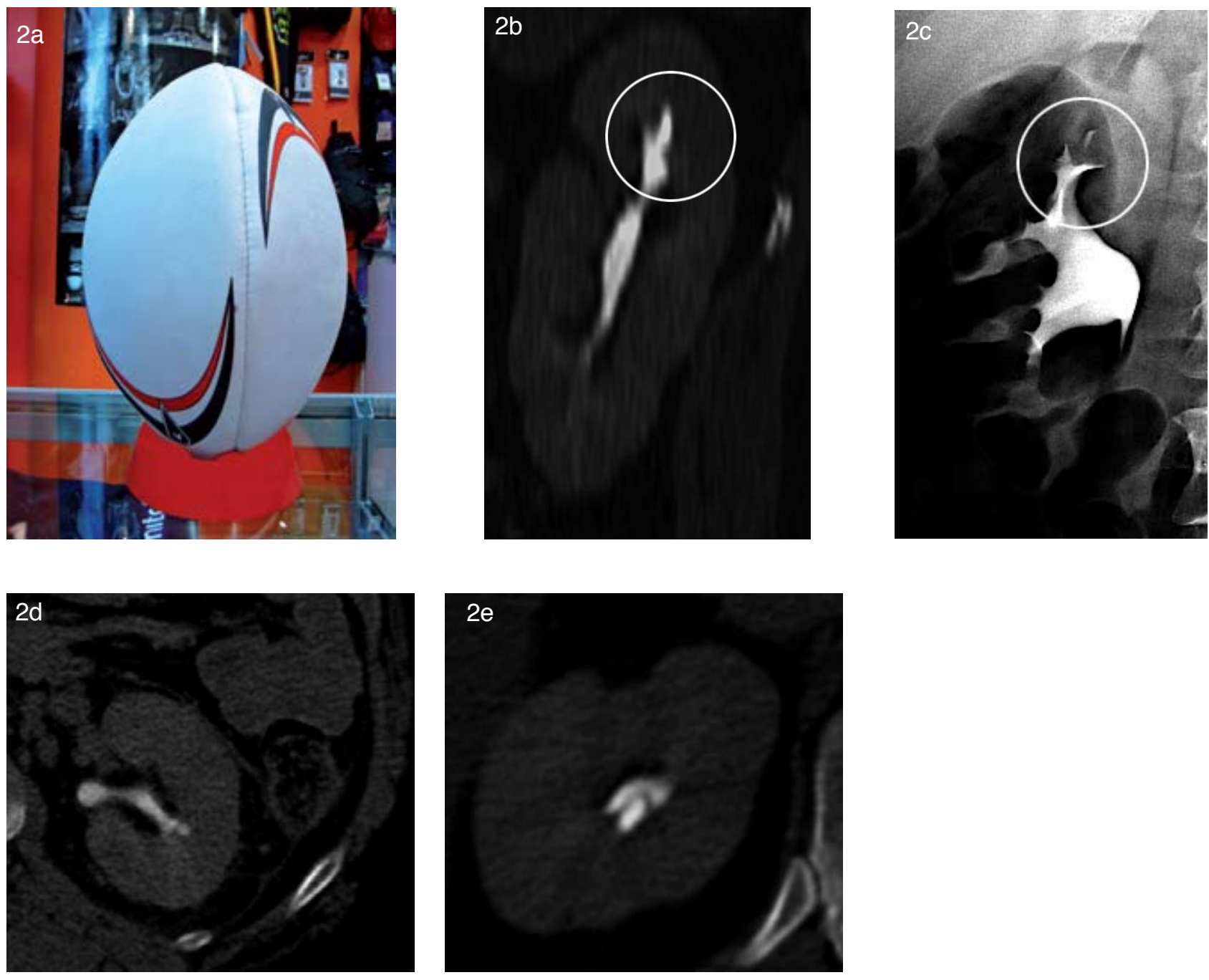

Figura 2. a) Balón de rugby en pedestal="ball in tee”. b) Necrosis papilar medular en TCMD, reconstrucción multiplanar sagital (círculo) y c) en urografía excretora (círculo). $d$-e) Similares hallazgos en reconstrucciones axiales de TCMD, en fase de eliminación.

El término descriptivo de la literatura anglosajona, "lobster claw" o tenaza de langosta, se refiere al patrón radiográfico de excavación fornicial papilar bilateral (Figura 3$)^{(1)}$.

- La forma in situ, en que no hay desprendimiento de la papila, lo que es casi indetectable en pielografía de eliminación. Siendo así, el destino de la punta de la papila, desprenderse pero permanecer dentro del cáliz excavado rodeado de contraste (dando el signo de la sombra en anillo o "signet ring"), reabsorberse totalmente, pasar al sistema colector (papila desprendida) o calcificar parcial o totalmente (nefrocalcinosis). La calcificación papilar puede ocurrir en cualquiera de los ejemplos previamente descritos, pudiendo presentar al menos dos tipos de morfologías: la nefropatía por analgésicos (Figura 4) y la calcificación triangular en anillo (o de la periferia de la papila necrótica); según algunos autores este último es un patrón de nefrocalcinosis, particularmente asociado a necrosis papilar ${ }^{(3,2,8)}$.

En las formas medular y papilar, la excavación puede ser extensa y producir una "papila desprendida" que al movilizarse hacia la pelvis renal origine sintomatología de cólico ureteral y hacerse visible en la urografía o en la fase excretora del UroTC como hidronefrosis y defectos de llene en la pelvis renal, que plantean los diagnósticos diferenciales con litiasis radiolúcida, bola fúngica o coágulos (Figura 5).

Cuando la papila se ha desprendido o reabsorbido deja una cavidad o un cáliz de aspecto sacular o bulboso, signo conocido en literatura anglosajona como "clubbed calix" (caliz en porra) (Figura 6)(9). Con el paso del tiempo el parénquima renal suprayacente (médula remanente + corteza) se atrofia, determinando un aspecto cicatricial a ese nivel(1). 

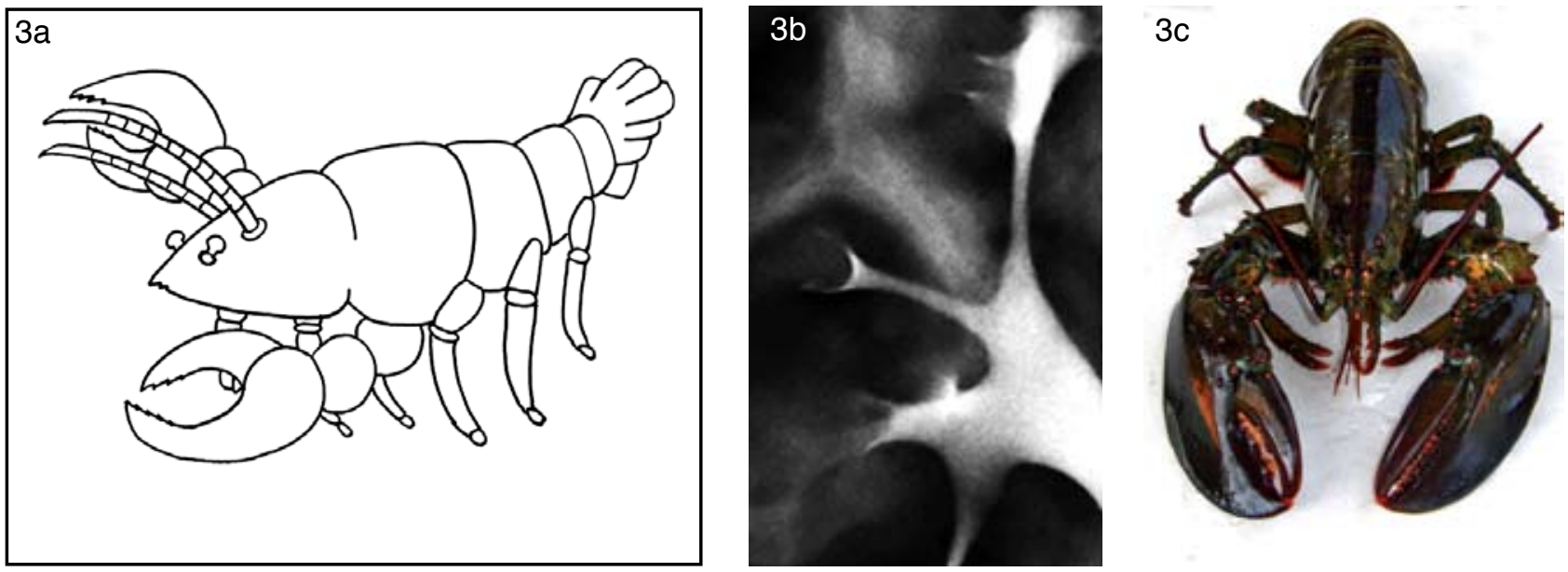

Figura 3. Necrosis papilar con forma de patrón en tenaza de langosta. Dibujo (a) grafica la característica tenaza de este animal que se reproduce en la urografía excretora (b). Homarus americanus o langosta americana (c), originaria de las aguas de América del Norte, con las típicas tenazas descritas. Cabe mencionar que las langostas chilenas (Jasus frontalis) carecen de este componente anatómico.

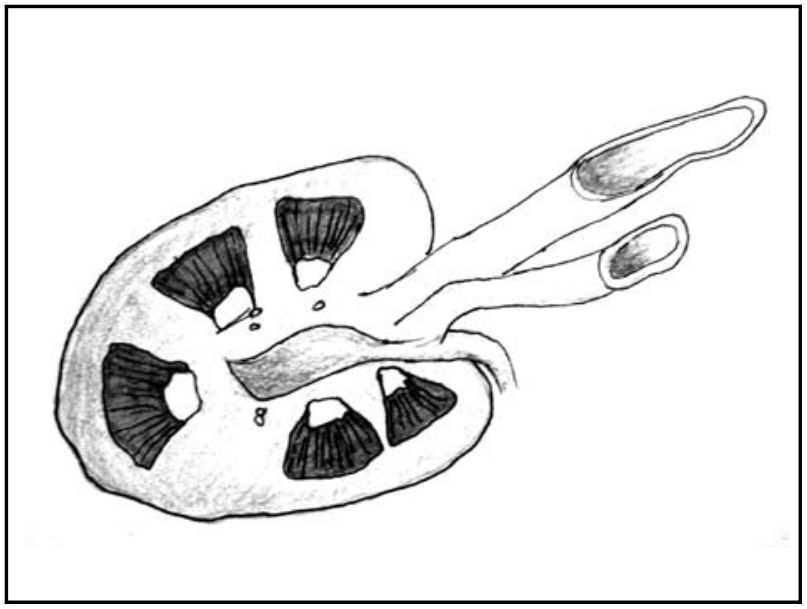

Figura 4. Patrón de calcificación de necrosis papilar (nefrocalcinosis medular) de la nefropatía por analgésicos que compromete las papilas medulares. Este signo puede presentarse tanto en necrosis papilar in situ (donde puede ser el único signo pielográfico) como en papilas desprendidas $^{(2,8)}$.

Figura 5 (a) y (b). Defecto de llene, rodeado de contraste, del grupo calicilar superior en un paciente con necrosis papilar conocida. En este caso se descartó la prescencia de litiasis en el estudio sin contraste, por lo que posiblemente este hallazgo corresponda a un coagulo o más probablemente a fragmentos papilares desprendidos de una necrosis central adyacente de morfología complementaria, que se alcanza a insinuar en (a).
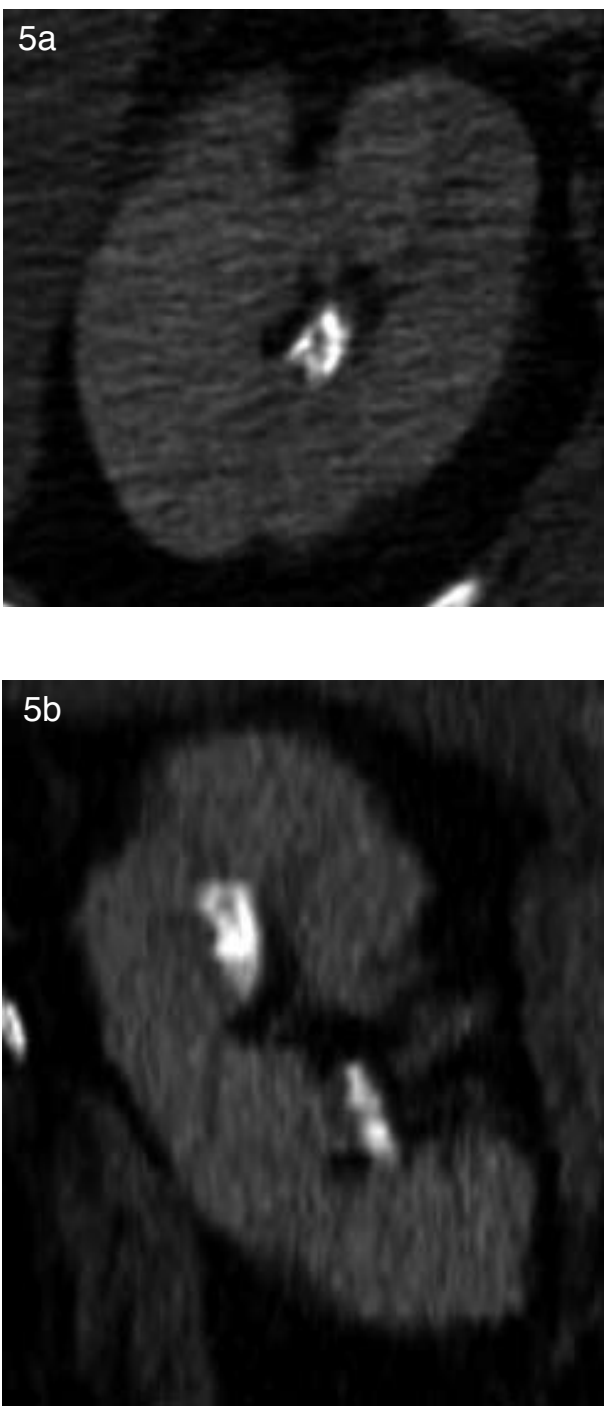

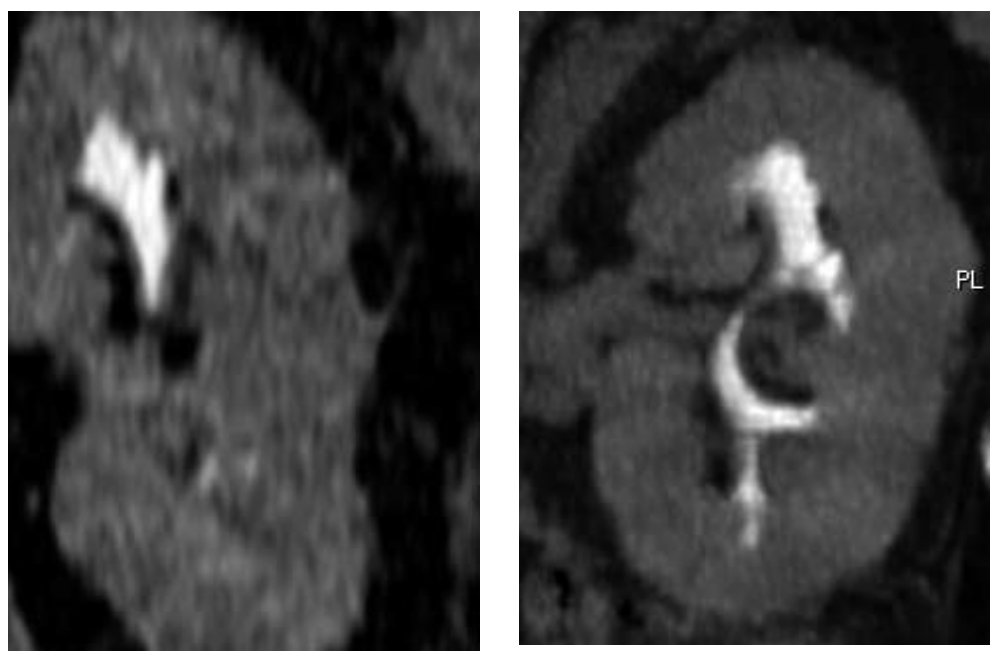

Figura 6 a y b. Patrón de necrosis papilar de cáliz en "porra" (polo superior). Nótese la excavación central en el polo inferior en (b).

\section{Diagnóstico diferencial}

En los casos de enfermedad avanzada con papilas desprendidas y también durante la fase de curación, en el área de las pirámides medulares se visualizan cavidades contiguas a los cálices en cuyo diagnóstico diferencial debieran ser consideradas otras causas de lesiones quísticas en estas mismas áreas o en el seno renal: hidronefrosis, megacálices congénitos, quistes parapiélicos o divertículos caliciales. Los megacálices congénitos constituyen una entidad rara, que debe ser diagnosticada después de descartar un cuadro obstructivo, previo o concurrente ${ }^{(2)}$.

La diferencia con la hidronefrosis debe ser establecida porque en esta entidad las "cavidades" en cuestión comprometen prácticamente todos los cálices (compromiso homogéneo y difuso) y en la necrosis papilar hay cálices afectados en distintos grados (presentando los patrones morfológicos ya descritos) alternando con otros sin afectación, asimetría propia de la necrosis ${ }^{(1,2,10)}$. Además, en la NP las cavidades presentan un contorno irregular, al contrario del contorno liso de la hidronefrosis y se extienden tan sólo hasta las arterias arcuatas (corteza renal)(1). Su diferenciación pudiera ser difícil en algunos pacientes con atrofia renal post-obstructiva o por reflujo, dado que en ellos también se observa atrofia papilar, secundaria a hiperpresión urinaria ${ }^{(2)}$.

El resto de las entidades mencionadas (quistes y divertículos) pueden ser diferenciadas por su ubicación, especialmente los segundos, en relación a los fórnices o infundíbulos ${ }^{(1)}$.

El diagnóstico diferencial en las formas calcificadas de necrosis papilar incluye otras causas de nefrocalcinosis medular (riñón en esponja medular, con su patrón en bouquet de flores) y la litiasis, en casos de papilas desprendidas ${ }^{(2)}$.

Aparte de los diagnósticos diferenciales mencionados, la tuberculosis renal es particularmente importante porque una de sus manifestaciones más precoces corresponde al de una NP difícilmente diferenciable del resto de las causas ya descritas ${ }^{(2)}$. En este contexto, el aspecto "apolillado" de las puntas papilares en la pielografía clásica no es tan específico y resulta difícil de distinguir de otras alteraciones ${ }^{(10)}$. Si bien se describe que las excavaciones tuberculosas periféricas tienen una extensión más profunda en la médula que las observadas en otro tipo de NP, la presencia de hallazgos asociados en el resto del sistema urinario (estenosis u obstrucción cicatricial infundibular y/o piélica con dilatación proximal secundaria o compromiso multifocal del tracto excretor urinario) son los parámetros en los que finalmente hay que apoyarse para fundamentar este diagnóstico(2,10).

\section{Cambios precoces en la necrosis papilar}

Una de las ventajas de la TCMD en relación con los métodos clásicos es la caracterización de los cambios isquémicos precoces. Estos no son visibles en la urografía excretora tradicional ni en la fase excretora de la TCMD, sino que se demuestran en la fase nefrográfica como pequeñas áreas mal definidas hipocaptantes en la punta de la pirámide medular.

Si el proceso isquémico medular es debido a un vasoespasmo y la circulación es restaurada dentro de un período razonable o si las condiciones predisponentes son corregidas, el tejido papilar afectado puede recuperarse ${ }^{(1,5)}$.

La pielonefritis focal y los abcesos renales son lesiones que pueden asemejarse y deben ser consideradas en el diagnóstico diferencial de estas alteraciones precoces. En estos casos, la base para establecer el diagnóstico diferencial es principalmente la ubicación y secundariamente los patrones de captación. La pielonefritis rara vez afecta la médula y habitualmente tiene un compromiso más bien lobar o segmentario, sin olvidar la presencia de captación periférica en fase venosa. Los abscesos suelen ser corticales o del 
límite córtico-medular y suelen presentar una pared captante, que no se observa en la NP(1).

\section{Conclusión}

La necrosis papilar es una entidad ampliamente conocida en la literatura radiológica clásica de origen multifactorial, con múltiples patrones de excavación visibles en la pielografía de eliminación y con un correlato morfológico muy parecido en la UroTC.

El diagnóstico precoz, con la visualización de hipocaptación medular y su diferenciación de otras entidades patológicas, es una de las principales innovaciones de este nuevo método de estudio, que a futuro podría mejorar el tratamiento y pronóstico de este tipo de pacientes.

\section{Agradecimientos}

A Carlos Orellana Seeger, (Langosta Lobster S.A.) por el acceso fotográfico a la langosta utilizada en este estudio y a Cristián Ramírez Pizarro por su colaboración en el procesamiento de imágenes.

\section{Bibliografía}

1. Jung DC, Kim SH, Jung SI, Hwang SI, Kim SH. Renal Papillary Necrosis: Review and Comparison of Findings at Multi-Detector Row CT and Intravenous Urography. RadioGraphics 2006; 26:1827-1836.

2. Davidson A. Enfermedades parenquimatosas con tamaño y contorno renal normales. In: Davidson's radiología del riñón, 3ra edición, Madrid. Editorial Marban. 2001: 327-356.

3. Albornoz JC. Efecto Secundario de los AINES: Revisión. Revista de la Sociedad Médico Quirúrgica del Hospital de Emergencia Pérez de León. 1997; 28(1): 48-54.

4. Rankin S. Renal Parenchymal Disease, including Renal Failure, Renovascular Disease and Transplantation. In: Adam A, Dixon AK, Grainger RG, Allison DJ, editors. Adam: Grainger \& Allison's Diagnostic Radiology, 5th ed. 2008, Elsevier Limited; chapter 39.

5. Lang EK, Macchia RJ, Thomas R, Davis R, RuizDeya G, Watson RA et al. Detection of medullary and papillary necrosis at an early stage by multiphasic helical computerized tomography. J Urol 2003; 170(1): 94-98.

6. Joffe SA, Servaes S, Okon S, Horowitz M. Multi-detector row CT urography in the evaluation of hematuria. Radiographics 2003; 23(6): 1441-1456.

7. Dyer RB, Chen MY, Zagoria RJ. Classic signs in uroradiology. Radiographics 2004; 24 Suppl 1: 247-280.

8. Federlene J. Section 3: Kidney and urinary tract. In: Federlene et al, Diagnostic imaging abdomen. First edition. Altona. Editorial Amirsys. 2005. pp:III-3-246, III-3-106.

9. Babaric Z. Fracaso renal. In: Barbaric Z. Radiología del aparato urinario. 2da edición, Madrid. Editorial Marban. 1995. pp251-267

10. Jander P. Capítulo 10: Enfermedades infecciosas del tracto urinario. In: Witten D et al (Eds). Urografía clínica, atlas y tratado de diagnóstico roentgenológico Tomo II. 1 Edición. Barcelona. Editorial Salvat.1983.pp 245378. 